NCU-HEP-k050

Aug 2012

rev. Jan 2013

ed. Mar. 2013

\title{
Majorana versus Dirac mass from holomorphic supersymmetric Nambu-Jona-Lasinio model
}

\author{
Yan-Min Dai \\ Department of Physics and Center for Mathematics and Theoretical Physics, \\ National Central University, Chung-Li, Taiwan 32054 \\ Gaber Faise * \\ Department of Physics and Center for Mathematics and Theoretical Physics, \\ National Central University, Chung-Li, Tawian 32054 and \\ Egyptian Center for Theoretical Physics, \\ Modern University for Information and Technology, Cairo, Egypt 11211 \\ Dong-Won Jungt \\ Department of Physics, National Tsing Hua University and \\ Physics Division, National Center for Theoretical Sciences, Hsinchu, Taiwan, 300 and \\ School of Physics, KIAS, Seoul, Korea 130-722 \\ Otto C. W. Kong \\ Department of Physics and Center for Mathematics and Theoretical Physics, \\ National Central University, Chung-Li, Tawian, 32054
}




\begin{abstract}
We study the theoretical features in relation to dynamical mass generation and symmetry breaking for the recently proposed holomorphic supersymmetric Nambu-Jona-Lasinio model. The basic model has two different chiral superfields (multiplets )with a strongly coupled dimension five foursuperfield interaction. In addition to the possibility of generation of Dirac mass between the pair established earlier, we show here the new option of generation of Majorana masses for each chiral superfield. We also give a first look at what condition may prefer Dirac over Majorana mass, illustrating that a split in the soft supersymmetry breaking masses is crucial. In particular, in the limit where one of the soft masses vanish, we show that generation of the Majorana mass is no longer an option, while the Dirac mass generation survives well. The latter is sensitive mostly to the average of the two soft masses. The result has positive implication on the application of the model framework towards dynamical electroweak symmetry breaking with Higgs superfields as composites.
\end{abstract}

\footnotetext{
*Electronic address: gfaisel@cc.ncu.edu.tw

${ }^{\dagger}$ Electronic address: dwjung@kias.re.kr

‡Electronic address: otto@phy.ncu.edu.tw
} 


\section{INTRODUCTION}

Dynamical mass generation and symmetry breaking are very interesting theoretical topics with important phenomenological applications. One of the simplest models of the kind is the Nambu-Jona-Lasinio (NJL) model [1]. It is also the first explicit model of spontaneous symmetry breaking. The analysis of the nonperturbative gap equation established that with a strong enough four-fermion interaction, a symmetry breaking Dirac fermion mass would result. When applied to the electroweak symmetry breaking of the Standard Model, it fails to give the relatively small experimental top quark mass [3]. Introducing heavier fourth family quarks to take the role of the top quark is essentially ruled out by other experimental constraints. The more interesting option of supplementing supersymmetry [4, 5] requires a too low $\tan \beta$ value to stay phenomenologically viable. The latter situation can be resolved with an alternative supersymmetrization of the NJL model recently proposed [6]. The version has a dimension five four-superfield interaction, which otherwise mimics well the most basic features of the NJL model.

Supersymmetry is an important theme in modern physics. One especially attractive feature, in our opinion, is that the scalar fields are now part of the chiral superfields with the chiral fermions. The chirality forbids any gauge invariant mass before breaking any symmetry. Moreover, the full matter (super)field spectrum is now strongly constrained by the gauge symmetry and their anomaly cancellation conditions. Introduction of the vectorlike pair of Higgs superfields with their unnatural gauge invariant mass in the usual formulation of the supersymmetric Standard Model looks particularly unattractive from the theoretical perspective. A NJL mechanism, with the Higgs superfield(s) generated as composite and the electroweak scale generated by strong dynamics is hence very appealing. The holomorphic supersymmetric Nambu-Jona-Lasinio model (HSNJL) ${ }^{1}$ construction [6, 6]

\footnotetext{
${ }^{1}$ In the first effort to produce a supersymmetric version of the NJL model, a dimension six four-superfield interaction was used [8]. It was found that soft supersymmetry breaking was needed for the kind of model to produce dynamical symmetry breaking and generate Dirac mass [8, [9]. The model, however, lost the basic NJL model feature of having a single auxiliary (super)field as both the (quark) composite and the symmetry breaking Higgs (super)field. The HSNJL model retains the latter feature, but it has a holomorphic (dimension five) four-superfield interaction which does not contain a four-fermion interaction. At least to the extent that a chiral superfield is the supersymmetrization of the fermion field, and the model has a four (quark) superfield interaction inducing a composite of two (quark) superfields with vacuum condensate to break symmetry and generate Dirac superfield(fermion) mass, we consider
} 
gives exactly such a scenario that looks compatible with all known experimental constraints. In Ref.[7], superfield gap equation analyses of the Dirac mass generation have been performed for the HSNJL model and the old supersymmetric model. Nontrivial symmetry breaking masses were established.

Distinguished from the old models, the HSNJL model is very rich in interesting theoretical features, some of which we report here. Firstly, the HSNJL model is also capable of generating Majorana masses of the chiral superfields. Note that the basic model has two superfields which could otherwise be the Dirac pair. In fact, in the generic case, the story of dynamical mass generation and hence the resulting symmetry breaking pattern becomes more complicated than the naive Dirac mass generation analysis would otherwise conclude. We present in this article an illustration of the Majorana mass generation-a feature that is unknown for models in the literature. Short of doing a comprehensive and fully generic gap equation analysis, we will compare the Majorana mass result here versus the Dirac mass result in our previous paper [7] to give a first answer to the competition of Majorana versus Dirac superfield masses. We will discuss how a splitting between the two input soft supersymmetry breaking masses favors the generation of Dirac superfield mass. In a very interesting particular case, we will show that at the limit, one of the soft masses vanish, the nontrivial Majorana mass would be killed as the nontrivial Dirac mass solution survives. Dynamical mass generation also implies dynamical symmetry breaking in general, as discussed in Ref.[7]. The result has an interesting implication to the application to electroweak symmetry breaking, though the focus of the present paper is on the theoretical features.

The details of the calculations involved are very similar to what we have presented in Ref.[7]. In the latter paper, we succeeded in getting the gap equations from first principle supergraph analyses both for the new HSNJL model and the old supersymmetric NJL model[8, 9] with the result of the latter case in perfect agreement with the one from the effective field theory analysis [9] and that of the simple NJL limit. For the interest of the general readers, we will only sketch the analyses here by highlighting only the essential features. Theorists interested in the details should also read carefully Ref.[7]. Further details together with a fully generic comprehensive analysis of the HSNJL model will be presented

it a supersymmetrization of the NJL model. Terminology aside, the physics features and their possible realization in nature are what is interesting. 
in a forthcoming publication [10]. In particular, note that the analyses rely heavily on the formulation of the generating functional, mass parameters, and self-energy amplitudes as superspace quantity as introduced in Ref.[7]. That is, only the full superspace analog of usual Minkowski spacetime field theory.

\section{DYNAMICAL GENERATION OF MAJORANA MASS}

The basic model has two different chiral superfields (multiplets) $\Phi_{+}$and $\Phi_{-}$, presumably carrying different quantum numbers. For instance, they may be different gauge multiplets. The dimension five four-superfield interaction is given by

$$
-\frac{G}{2} \int d^{4} \theta \Phi_{+} \Phi_{+} \Phi_{-} \Phi_{-}\left(1+B \theta^{2}\right) \delta^{2}(\bar{\theta})
$$

It is really a superpotential term, as indicated by the $\delta^{2}(\bar{\theta})$, hence holomorphic. In our earlier works [6, 7], the possibility of superfield condensate $\left\langle\Phi_{+} \Phi_{-}\right\rangle$giving rise to a Dirac mass term $\mathcal{M} \Phi_{+} \Phi_{-}$has been investigated. Unlike the dimension six four-supefield interaction, the superpotential term offers also the option of, say, a condensate of $\left\langle\Phi_{+} \Phi_{+}\right\rangle$or $\left\langle\Phi_{-} \Phi_{-}\right\rangle$giving Majorana mass terms $\mathcal{M}_{-} \Phi_{-} \Phi_{-}$and $\mathcal{M}_{+} \Phi_{+} \Phi_{+}$, respectively. To investigate the option, we proceed similar to the Dirac mass analysis [7] by deriving the gap equations for $\mathcal{M}_{+}$and $\mathcal{M}_{-}$.

Consider the Lagrangian density written as

$$
\begin{aligned}
\mathcal{L}= & \int d^{4} \theta\left[\Phi_{+}^{\dagger} \Phi_{+}\left(1-\Delta_{+}\right)+\Phi_{-}^{\dagger} \Phi_{-}\left(1-\Delta_{-}\right)\right. \\
& \left.+\left(\mathcal{M}_{+} \Phi_{+} \Phi_{+} \delta^{2}(\bar{\theta})+\mathcal{M}_{-} \Phi_{-} \Phi_{-} \delta^{2}(\bar{\theta})+H . c .\right)\right]+\mathcal{L}_{I}
\end{aligned}
$$

with

$$
\mathcal{L}_{I}=\int d^{4} \theta\left[-\mathcal{M}_{+} \Phi_{+}^{a i} \Phi_{+}^{b i}-\mathcal{M}_{-} \Phi_{-}^{\bar{a} j} \Phi_{-}^{\bar{b} j}-\frac{G}{2} \Phi_{+}^{a i} \Phi_{+}^{b i} \Phi_{-}^{\bar{a} j} \Phi_{-}^{\bar{b} j}\left(1+B \theta^{2}\right)\right] \delta^{2}(\bar{\theta})+H . c .
$$

where we consider explicitly $S U\left(N_{c}\right) \otimes S O\left(N_{r}\right)$ multiplets $\Phi_{+}^{a i}$ and $\Phi_{-}^{\bar{a} j}$ in the fundamental and its conjugate representations of $S U\left(N_{c}\right)$ as indicated by the $a$ and $\bar{a}$ (as well as $b$ and $\bar{b})$ indices, ${ }^{2}$ respectively, and the real fundamental representation of $S O\left(N_{r}\right)$ as indicated

\footnotetext{
${ }^{2}$ Note that we use here the $\bar{a}$ upper index in place of a lower $a$ index with the summation convention contracting it with the upper $a$ index.
} 
by the $i$ and $j$ indices. Moreover, in the first part of the Lagrangian, we have suppressed all indices for simplicity. The same applies to the expressions below; the indices are not shown unless necessary. Note that the dimension five interaction is invariant under the symmetry, while the Majorana mass terms break the $S U\left(N_{c}\right)$ symmetry. For a clear comparison, the Dirac mass term $\mathcal{M} \Phi_{+}^{a i} \Phi_{-}^{\bar{a} j}$ preserves $S U\left(N_{c}\right)$ symmetry while generally breaking the $S O\left(N_{r}\right)$ symmetry.

Here, $\Delta_{ \pm}=\tilde{m}_{ \pm}^{2} \theta^{2} \bar{\theta}^{2}$ characterizes the input soft supersymmetry breaking mass-squared $\tilde{m}_{ \pm}^{2}$ for the corresponding scalar field $A_{ \pm}$and $\mathcal{M}_{ \pm}$superfield Majorana mass parameter

$$
\mathcal{M}_{ \pm}=m_{ \pm}-\theta^{2} \eta_{ \pm}
$$

with the supersymmetric Majorana mass $m_{ \pm}$and its supersymmetry breaking counterpart $\eta_{ \pm}$. The mass parameters in the above equation are what we aim at generating dynamically. The gap equations are given by

$$
-\mathcal{M}_{ \pm}=\left.\Sigma_{ \pm \pm}^{(\text {loop })}\left(p, \theta^{2}\right)\right|_{\text {on shell, }}
$$

where $\Sigma_{ \pm \pm}^{(\text {loop })}$ denotes the lowest order contributions to the proper self-energy from loop diagrams involving the four-superfield interactions. Note that $\Sigma_{++}^{(l o o p)}$ has contribution involving the $\Phi_{-}$superfield propagator $\left\langle T\left(\Phi_{-}(1) \Phi_{-}(2)\right)\right\rangle$, and $\Sigma_{--}^{(l o o p)}$ the propagator $\left\langle T\left(\Phi_{+}(1) \Phi_{+}(2)\right)\right\rangle$. The propagator should include the Majorana masses $\mathcal{M}_{ \pm}$dependence. The propagators are given in the same form as the Dirac case of $\left\langle T\left(\Phi_{+}(1) \Phi_{-}(2)\right)\right\rangle$, namely, as

$$
\begin{gathered}
\left\langle T\left(\Phi_{ \pm}(1) \Phi_{ \pm}(2)\right)\right\rangle=\frac{i \bar{m}_{ \pm}}{p^{2}\left(p^{2}+\left|m_{ \pm}\right|^{2}\right)} \frac{D_{1}^{2}}{4} \delta_{12}^{4} \\
-\frac{i}{\left[\left(p^{2}+\left|m_{ \pm}\right|^{2}+\tilde{m}_{ \pm}^{2}\right)^{2}-\left|\eta_{ \pm}\right|^{2}\right]}\left[\frac{\bar{\eta}_{ \pm} D_{1}^{2} \bar{\theta}_{1}^{2}}{4}-\frac{\eta_{ \pm}\left|m_{ \pm}\right|^{2} D_{1}^{2} \theta_{1}^{2}}{4 p^{2}}\right] \delta_{12}^{4} \\
+\frac{i \bar{m}_{ \pm}\left[\tilde{m}_{ \pm}^{2}\left(p^{2}+\left|m_{ \pm}\right|^{2}+\tilde{m}_{ \pm}^{2}\right)-\left|\eta_{ \pm}\right|^{2}\right]}{\left(p^{2}+\left|m_{ \pm}\right|^{2}\right)\left[\left(p^{2}+\left|m_{ \pm}\right|^{2}+\tilde{m}_{ \pm}^{2}\right)^{2}-\left|\eta_{ \pm}\right|^{2}\right]}\left[\frac{D_{1}^{2} \theta_{1}^{2} \bar{\theta}_{1}^{2}}{4}+\frac{\bar{\theta}_{1}^{2} \theta_{1}^{2} D_{1}^{2}}{4}\right] \delta_{12}^{4} .
\end{gathered}
$$

We have the gap equations

$$
\begin{aligned}
m_{ \pm} & =\frac{\bar{\eta}_{\mp} G N_{r}}{2} I_{2}\left(\left|m_{\mp}\right|^{2}, \tilde{m}_{\mp}^{2},\left|\eta_{\mp}\right|, \Lambda^{2}\right), \\
\eta_{ \pm} & =\bar{m}_{\mp} G N_{r} I_{1}\left(\left|m_{\mp}\right|^{2}, \tilde{m}_{\mp}^{2},\left|\eta_{\mp}\right|, \Lambda^{2}\right)-\frac{\bar{\eta}_{\mp} G B N_{r}}{2} I_{2}\left(\left|m_{\mp}\right|^{2}, \tilde{m}_{\mp}^{2},\left|\eta_{\mp}\right|, \Lambda^{2}\right),
\end{aligned}
$$

where $I_{1}\left(|m|^{2}, \tilde{m}^{2},|\eta|, \Lambda^{2}\right)$ and $I_{2}\left(|m|^{2}, \tilde{m}^{2},|\eta|, \Lambda^{2}\right)$ are the same loop integrals as before [7], the details of which we will discuss below. 
The first thing to note from the gap equation results is that they are almost of exactly the same form as the Dirac case [7]. Actually, if we take identical soft masses $\tilde{m}_{ \pm}^{2}=\tilde{m}^{2}$, we have obviously a symmetric solution relative to $\Phi_{+}$and $\Phi_{-}$, which is exactly the same as the gap equation for the Dirac mass case shown in Ref. [7] (with $N_{r}$ replaced by $N_{c}$ ). For instance, considering only the case of real values for $m_{ \pm}$and $\eta_{ \pm}$under the assumption of a real and small $B$ value, we find that a nontrivial solution exists for large enough $G$ (taken as real and positive here by convention) satisfying

$$
G>\sqrt{G_{0}^{2}+b^{2}}+b \sim G_{0}+b,
$$

where

$$
G_{0}^{2}=\frac{512 \pi^{2}}{\tilde{m}^{2} \ln \left(1+\frac{\Lambda^{2}}{\tilde{m}^{2}}\right)\left[\ln \left(1+\frac{\Lambda^{2}}{\tilde{m}^{2}}\right)-\frac{\Lambda^{2}}{\tilde{m}^{2}+\Lambda^{2}}\right]}
$$

gives the critical $G^{2}$ for $B=0$, and

$$
b=B \frac{8 \pi^{2}}{\tilde{m}^{2} \ln \left(1+\frac{\Lambda^{2}}{\tilde{m}^{2}}\right)} .
$$

Details are given in Appendix A in Ref.[7]. Note that $B$ may be positive or negative, or more generally contains a complex phase. The solution condition for more general cases is to be further investigated. So, a nontrivial Majorana masses solution is possible, or as likely as that of the case for the Dirac mass generation.

\section{DIRAC VERSUS MAJORANA MASSES}

While the above analysis established the holomorphic four-superfield interaction as being capable of dynamically generating superfield Majorana masses, the result can put the Dirac mass generation scenario under question. After all, true Dirac mass means a nonzero $\mathcal{M} \Phi_{+} \Phi_{-}$mass term without $\mathcal{M}_{+}$and $\mathcal{M}_{-}$. It is important to note that the mass terms that arise have direct implications on the resultant symmetry breaking pattern. In the explicit example of the $\Phi_{+}^{a i}$ and $\Phi_{-}^{\bar{a} j}$ multiplet illustrated above, we can see that the $\mathcal{M}_{+}$and $\mathcal{M}_{-}$mass terms and the Dirac mass term $\mathcal{M}$ break different parts of the symmetries in the Lagrangian. For example, in the application to the electroweak symmetry breaking [6, 7], any Majorana mass term would be breaking the color symmetry. A fully general analysis considering a generic mass matrix for the $\Phi_{+}$and $\Phi_{-}$superfields may have to be performed 
to answer the important question [10] of under what condition will the interaction give rise to any particular symmetry breaking pattern. However, it is interesting to see that with the gap equation results we have so far, one can get a strong indication of how a pure Dirac mass generation may be obtained. The bottom line is that a split in soft masses favors Dirac mass generation over Majorana mass generation. Let us illustrate the story.

We note in passing that the gap equation result involves a $1 / N$ approximation, with $N$ being $N_{c}$ for the Dirac case and $N_{r}$ for the Majorana case, and there is a technical complication at the limit where the approximation is no good or invalid. It will be interesting to see if a gap equation can be obtained for the case $N_{r}=1$. It is reasonable to think that the mass generation and symmetry breaking mechanism still works in the case for a sufficiently strong interaction. We will get back to discussing the $N_{r}$ and $N_{c}$ dependence issue and its implication further at the end of Sec. IV.

In the discussion below, we will compare the gap equation results for the Majorana mass generations discussed above, i.e., assuming no off-diagonal $\mathcal{M} \Phi_{+} \Phi_{-}$mass term, and that of the (pure) Dirac mass generation analysis of Ref.[7] where there is also the hidden assumption of no diagonal (Majorana) masses $\mathcal{M}_{+}$and $\mathcal{M}_{-}$. This is not the full rigorous way to address the question of what would be the resultant mass matrix of $\Phi_{+}$and $\Phi_{-}$, but we can at least give some insight into some qualitative aspect of the question.

We are focusing here on the effect of the soft masses parameters on the symmetry breaking. The more trivial effect of the $N_{r}$ and $N_{c}$ values is neglected for the moment. To be exact, one may consider us as taking $N_{r}=N_{c}=N$ and rewriting $G N$ as simply $G$ below.

As in the above discussions on the Majorana mass case, we focus on the simpler case with $B=0$. Eliminating $G$ ( i.e., $G N_{r}$ ) from the gap equations, we get the relations

$$
2\left|m_{ \pm}\right|^{2} I_{1}\left(\left|m_{ \pm}\right|^{2}, \tilde{m}_{ \pm}^{2},\left|\eta_{ \pm}\right|, \Lambda^{2}\right)=\left|\eta_{\mp}\right|^{2} I_{2}\left(\left|m_{\mp}\right|^{2}, \tilde{m}_{\mp}^{2},\left|\eta_{\mp}\right|, \Lambda^{2}\right) .
$$


The integrals are given by ${ }^{3}$

$$
\begin{gathered}
I_{1}\left(|m|^{2}, \tilde{m}^{2},|\eta|, \Lambda^{2}\right)=\frac{1}{16 \pi^{2}}\left[\frac{1}{2}\left(|m|^{2}+\tilde{m}^{2}\right) \ln \frac{\left(\Lambda^{2}+|m|^{2}+\tilde{m}^{2}\right)^{2}-|\eta|^{2}}{\left(|m|^{2}+\tilde{m}^{2}\right)^{2}-|\eta|^{2}}\right. \\
\left.-|m|^{2} \ln \frac{\left(\Lambda^{2}+|m|^{2}\right)}{|m|^{2}}+\frac{|\eta|}{2} \ln \frac{\left(\Lambda^{2}+|m|^{2}+\tilde{m}^{2}+|\eta|\right)\left(|m|^{2}+\tilde{m}^{2}-|\eta|\right)}{\left(\Lambda^{2}+|m|^{2}+\tilde{m}^{2}-|\eta|\right)\left(|m|^{2}+\tilde{m}^{2}+|\eta|\right)}\right] \\
I_{2}\left(|m|^{2}, \tilde{m}^{2},|\eta|, \Lambda^{2}\right)=\frac{1}{32 \pi^{2}}\left[\ln \frac{\left(\Lambda^{2}+|m|^{2}+\tilde{m}^{2}\right)^{2}-|\eta|^{2}}{\left(|m|^{2}+\tilde{m}^{2}\right)^{2}-|\eta|^{2}}\right. \\
\left.+\frac{|m|^{2}+\tilde{m}^{2}}{|\eta|} \ln \frac{\left(\Lambda^{2}+|m|^{2}+\tilde{m}^{2}+|\eta|\right)\left(|m|^{2}+\tilde{m}^{2}-|\eta|\right)}{\left(\Lambda^{2}+|m|^{2}+\tilde{m}^{2}-|\eta|\left(|m|^{2}+\tilde{m}^{2}+|\eta|\right)\right)}\right] .
\end{gathered}
$$

On the $|m|-|\eta|$ plane, the $I_{2}$ expression is positive definite while the $I_{1}$ expression has maximum value at the origin given by $\frac{\tilde{m}^{2}}{16 \pi^{2}} \ln \frac{\left(\Lambda^{2}+\tilde{m}^{2}\right)}{\tilde{m}^{2}}$, which implies that $I_{1}$ will always be negative for $\tilde{m}^{2}=0$. What is interesting then is that in the case where one of the soft masses vanish, say, $\tilde{m}_{+}^{2}=0,\left|m_{+}\right|$and $\left|\eta_{-}\right|$will then be forced to vanish for the above relation to be satisfied. But the tachyonic bound for $\left|\eta_{+}\right|\left[\left|\eta_{ \pm}\right|<\left(|m|^{2}+\tilde{m}_{ \pm}^{2}\right) / 2\right.$ for the Majorana case] will then force it to vanish, hence, giving also $\left|m_{-}\right|=0$. That is independent of the value of the coupling.

The argument above shows there will be no nontrivial Majorana masses generated in the case in which one of the soft masses vanish. Vanishing, common, soft masses give no dynamical Dirac mass either [7]. In general, it is easy to see that smaller soft supersymmetry breaking mass disfavors the dynamical mass generations, requiring a strong coupling $G$ to achieve it, as also explicitly illustrated by the expression for the $G$ threshold given in Ref.[7]. However, when there is a splitting between the soft masses of the two superfields, there is a crucial difference between the Majorana mass and Dirac mass cases. While the supersymmetric part $m$ of each Majorana mass is directly sensitive to the vanishing of the corresponding soft mass, the Dirac mass result is more sensitive to the average of the two soft masses [7]. We have seen that when one $m$ vanishes, it implies that all Majorana mass parameters vanish, at least for $B=0$. We will show explicitly in the next section that having one vanishing soft mass does not adversely affect the dynamical generation of Dirac mass. Having one small soft mass decreases the possibility of Majorana mass generation, pushing up the threshold coupling, but has limited effect on the Dirac mass generation.

\footnotetext{
${ }^{3}$ The expression is formally equivalent to the earlier form given in Ref.[7], where the $\tanh ^{-1}$ function is involved, which, straightly speaking, has a domain of definition problem.
} 


\section{DIRAC MASS GENERATION WITH ONE OF THE SOFT MASSES VAN- ISHING}

The gap equation for the case of Dirac mass generation with two different soft masses has essentially been given in Ref.[7] (see Appendix B). We have (with $G$ in place of $G N_{c}$ )

$$
\begin{aligned}
m & =\frac{\bar{\eta} G}{2} I_{2}^{\prime}\left(|m|^{2}, \tilde{m}_{+}^{2}, \tilde{m}_{-}^{2},|\eta|, \Lambda^{2}\right) \\
\eta & =\bar{m} G I_{1}^{\prime}\left(|m|^{2}, \tilde{m}_{+}^{2}, \tilde{m}_{-}^{2},|\eta|, \Lambda^{2}\right)-\frac{\bar{\eta} G B}{2} I_{2}^{\prime}\left(|m|^{2}, \tilde{m}_{+}^{2}, \tilde{m}_{-}^{2},|\eta|, \Lambda^{2}\right)
\end{aligned}
$$

where $I_{1}^{\prime}$ and $I_{2}^{\prime}$ are the loop integrals. The expressions of the integrals as given in Ref.[7],

however, have some typos and have not been written in the best form. The integral should be exactly

$$
\begin{aligned}
& I_{1}^{\prime}\left(|m|^{2}, \tilde{m}_{+}^{2}, \tilde{m}_{-}^{2},|\eta|, \Lambda^{2}\right)=2 \int \frac{d^{4} k}{(2 \pi)^{4}} \frac{\left(\frac{\tilde{m}_{+}^{2}+\tilde{m}_{-}^{2}}{2}\right)\left(k^{2}+|m|^{2}+\frac{\tilde{m}_{+}^{2}+\tilde{m}_{-}^{2}}{2}\right)-\left(\frac{\tilde{m}_{+}^{2}-\tilde{m}_{-}^{2}}{2}\right)^{2}-|\eta|^{2}}{\left(k^{2}+|m|^{2}\right)\left[\left(k^{2}+|m|^{2}+\frac{\tilde{m}_{+}^{2}+\tilde{m}_{-}^{2}}{2}\right)^{2}-\left(\frac{\tilde{m}_{+}^{2}-\tilde{m}_{-}^{2}}{2}\right)^{2}-|\eta|^{2}\right]}, \\
& I_{2}^{\prime}\left(|m|^{2}, \tilde{m}_{+}^{2}, \tilde{m}_{-}^{2},|\eta|, \Lambda^{2}\right)=\int \frac{d^{4} k}{(2 \pi)^{4}} \frac{1}{\left[\left(k^{2}+|m|^{2}+\frac{\tilde{m}_{+}^{2}+\tilde{m}_{-}^{2}}{2}\right)^{2}-\left(\frac{\tilde{m}_{+}^{2}-\tilde{m}_{-}^{2}}{2}\right)^{2}-|\eta|^{2}\right]} .
\end{aligned}
$$

What we failed to highlight in that paper are the relations

$$
\begin{aligned}
& I_{1}^{\prime}\left(|m|^{2}, \tilde{m}_{+}^{2}, \tilde{m}_{-}^{2},|\eta|, \Lambda^{2}\right)=I_{1}\left(|m|^{2}, \tilde{m}_{a v}^{2},\left|\eta^{\prime}\right|, \Lambda^{2}\right), \\
& I_{2}^{\prime}\left(|m|^{2}, \tilde{m}_{+}^{2}, \tilde{m}_{-}^{2},|\eta|, \Lambda^{2}\right)=I_{2}\left(|m|^{2}, \tilde{m}_{a v}^{2},\left|\eta^{\prime}\right|, \Lambda^{2}\right),
\end{aligned}
$$

where

$$
\tilde{m}_{a v}^{2}=\frac{\tilde{m}_{+}^{2}+\tilde{m}_{-}^{2}}{2}
$$

is the average value of the soft masses and

$$
\left|\eta^{\prime}\right|=\sqrt{|\eta|^{2}+\left(\frac{\tilde{m}_{+}^{2}-\tilde{m}_{-}^{2}}{2}\right)^{2}} .
$$

The relations are actually easy to appreciate from the physics point of view by comparing how the various parameters go into the (scalar) mass eigenvalues in the cases with different or identical soft masses.

From the above, one can easily use the properties of the $I_{1}$ and $I_{2}$ integrals to look at the case of Dirac mass generation with only one of the soft masses vanishing. For example, one 


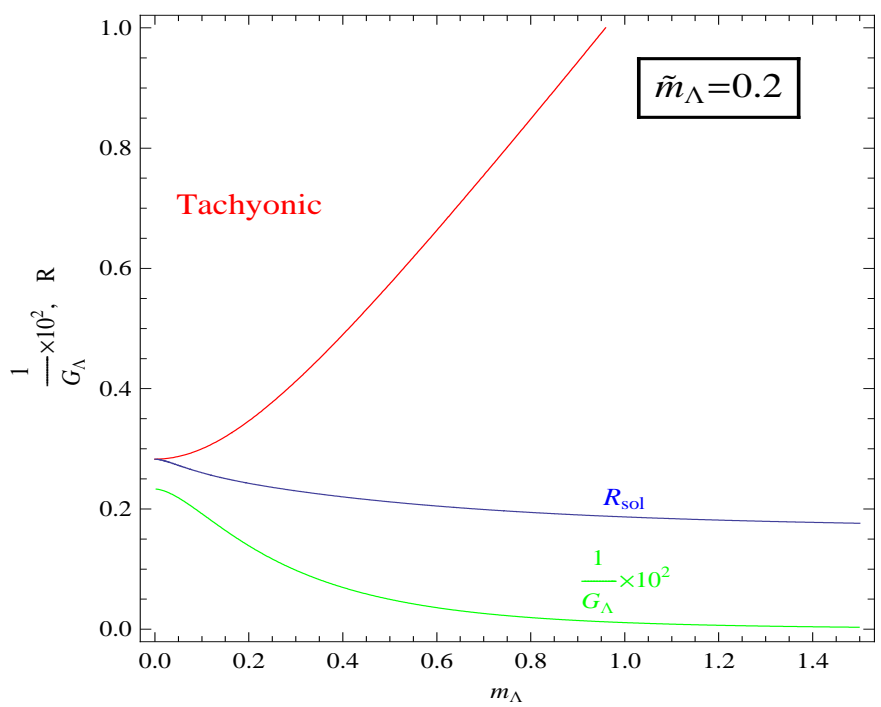

FIG. 1: Illustrative numerical plot of the solution curve on the $R_{\Lambda}-m_{\Lambda}$ plane. $R_{\Lambda}$ and $m_{\Lambda}$ are corresponding dimensionless parameters for $R=|\eta| /|m|$ and $|m|$ normalized to the basic (cutoff) scale $\Lambda$. Likewise, $\tilde{m}_{\Lambda}^{2}=\tilde{m}_{a v}^{2} / \Lambda^{2}$. The value of $1 / G_{\Lambda}$ versus $m_{\Lambda}, G_{\Lambda}=|G| \Lambda$ being the normalized coupling, is also given.

can check that since $I_{1}$ increases as $|m|$ and $\left|\eta^{\prime}\right|$ decrease, it attains a maximum at $|m|=0$ and $\left|\eta^{\prime}\right|$ minimum of $\left|\frac{\tilde{m}_{+}^{2}-\tilde{m}_{-}^{2}}{2}\right|$ which corresponds to $|\eta|=0$, and the maximum value is given by exactly the same expression as before, namely, $\frac{2 \tilde{m}_{a v}^{2}}{16 \pi^{2}} \ln \frac{\Lambda^{2}+2 \tilde{m}_{a v}^{2}}{2 \tilde{m}_{a v}^{2}}$. So, twice the average soft mass here, which equals the single nonzero soft mass, plays the role of the single soft mass relevant for the particular individual Majorana mass equation or the universal soft mass case for the Majorana as well as Dirac mass generation analysis. The simple conclusion is that having one vanishing soft mass does not kill the Dirac mass generation as it does to the Majorana mass generations.

A further look at the solution curve as given by Eq.(10) of this Dirac case, $\frac{\bar{\eta}}{2 m} I_{2}=\frac{\bar{m}}{\eta} I_{1}(=$ $\frac{1}{G}$ ), on the plane of $R=|\eta| /|m|$ versus $|m|$ (for fixed $\Lambda$ and $\tilde{m}_{a v}^{2}$ ) is particularly illustrative. A case example is plotted in Fig. 1. It should be noted that $R$ has mass dimension one while $\eta$ has mass dimension two. In general, $R$ decreases monotonically with $|m|$, slowing down to an asymptotical constant value at the $|m| \rightarrow \infty$ limit. On the physics side, it is sensible to restrict all mass parameters not to go beyond the scale of order $\Lambda$, which is 
the model cutoff scale. The asymptotical analysis helps though to show the mathematical features. A careful calculation of the limiting expressions for $I_{1}$ and $I_{2}$ gives the asymptotic value as $R_{\infty}=\sqrt{\frac{2 \tilde{m}_{a v}^{2}}{3}}$, a constant. For the $|m| \rightarrow 0$ limit, the limiting expressions for $I_{1}$ and $I_{2}$ give $I_{1}=\tilde{m}_{a v}^{2} I_{2}$, hence, the constant $R_{o}=\sqrt{2 \tilde{m}_{a v}^{2}}$. The latter is right at the boundary of the inadmissible tachyonic region (for lighter scalar mass eigenstate from $A_{ \pm}$). The bound itself is given by $R_{t}=\sqrt{2 \tilde{m}_{a v}^{2}+|m|^{2}}$ increasing with $|m|$. The solution curve is safely below the tachyonic bound. One can also get the asymptotic expressions for the coupling $G$, or rather $|G|$. We have $\left|G_{\infty}\right|=32 \sqrt{6} \pi^{2}|m|^{4} / \tilde{m}_{a v} \Lambda^{4}$, and the threshold coupling $\left|G_{o}\right|=16 \sqrt{2} \pi^{2}\left[\tilde{m}_{a v} \ln \left(1+\frac{\Lambda}{2 \tilde{m}_{a v}^{2}}\right)\right]^{-1}$.

Before we conclude, let us look a bit more into the question about the $N_{c}$ and $N_{r}$ values. For that matter, let us take the case of identical soft supersymmetry breaking masses. What is really relevant here is the number of (color) states involved in the $\Phi_{+}^{a i}$ and $\Phi_{-}^{\bar{a} j}$ pair we simply denote by $N_{c}$ and the numbers of states involved in $\Phi_{+}^{a i} \Phi_{+}^{b i}$ and $\Phi_{-}^{a j} \Phi_{-}^{b j}$. $G N_{c}$ is the is the "coupling" parameter that shows up in the gap equation for Dirac mass $\mathcal{M}$ while the last two, say, $N_{+}$and $N_{-}$, show up in the place of $N_{c}$ in the gap equations for $\mathcal{M}_{-}$and $\mathcal{M}_{+}$, respectively. Note that $N_{+}$and $N_{-}$may in general be different, say involving different symmetries like $S O\left(N_{+}\right)$and $S O\left(N_{-}\right)$, or in different representations of one $S O\left(N_{r}\right)$. For $N_{+} \neq N_{-}$, the Majorana mass generation will be constrained by the smaller $N_{ \pm}$value, as one cannot have a nontrivial solution to only one of the two masses. But the mass values will have the $N_{+}$and $N_{-}$ratio. In both the Dirac and Majorana cases, it is clear that as the strong coupling condition is on the $G N$ product, a large $N$ makes the dynamical generation of the relevant mass term easier. A coupling value $G$ large enough, for instance, for $G N_{c}$ to be above the critical limit but have $G N_{r}$ below the limit will be expected to give pure Dirac mass. The situation reverses with $N_{r}>N_{c}$.

The case for $N=1$ is special in the sense that the $1 / N$ approximation behind the gap equation derivation is completely not justified. More effort may be needed to obtain a useful gap equation for the case. Even so, an analysis equivalent to taking the exact gap equation here is common in the literature, starting from the quenched planar approximation of QED by Bardeen et.al. [11]. Qualitatively, it is reasonable to believe that the dynamical mechanism still works for large enough $G$. And at least in the case that the dynamical mechanism works, the interesting feature of Dirac mass generation being sensitive mostly to the average of the two soft masses while Majorana masses to the smaller soft mass between 
the two will very likely survive.

\section{CONCLUSIONS}

We show that the HSNJL model is capable of dynamically generating Majorana masses for the two superfields involved. This is an important alternative to the Dirac mass generation analyzed earlier. The general question of under what condition a particular mass pattern, or mass matrices, for the two superfields will result becomes an important question to address theoretically. It also has a phenomenological implication on whether there is a successful application of the model to electroweak symmetry breaking. We give here a first answer to the complicated question, that a splitting in the input soft supersymmetry breaking masses favors Dirac over Majorana mass. In particular, in the limit where one soft mass vanishes, nontrivial Majorana mass is not possible while Dirac mass can still be generated. Hence, one expects that a strong coupling within a particular range dictated by the different soft masses' values will give a symmetry breaking answer that corresponds to the pure Dirac mass.

In the case of the application to the electroweak symmetry breaking [6, 7], the model has a four-superfield interaction involving three different gauge multiplets and hence three soft masses of the top and bottom squark sector. It has already been noted that a split in the soft masses between the two sectors at least will be needed to give the phenomenologically required top and bottom mass ratio. It is encouraging to see that the kind of mass splitting also disfavors the generation of Majorana masses for the quark superfield multiplets, which of course will break color and electric charge symmetry. We note also that the strong QCD attraction will also play an important role there favoring color singlet vacuum condensates. Full details of all those await further analysis. The most tricky part is the fact that $N_{r}=1$ is involved. However, we consider it reasonable to claim, from what we have been able to establish so far, that the HSNJL stands qualitatively viable as a model for electroweak symmetry breaking. We hope to be able to present in a future publication full quantitative results.

Finally, we want to comment on the perspective of using an effective field theory approach to look at the symmetry breaking. Assuming a certain auxiliary Higgs (super)field formation as a composite, one can write down the effective field theory on the kind of model [6, 7, 9]. 
An (infinite) wave functional renormalization makes the Higgs (super)field dynamical at low energy. One can apply an analysis of the effective field theory to find the symmetry breaking solution. This was what was done in Ref. [9] for the case of the dimension six four-superfield interaction. The gap equation approach accomplished in Ref.[7], in comparison, can be considered as deriving instead of assuming the composite formation. It gives the nontrivial mass, hence, symmetry breaking solution, without putting in any composite structure. The composite formation as Higgs includes its role in having mass-generating nontrivial vacuum. For the old model, we achieve that and verify the explicit results of Ref. [9]. For the HSNJL model, we derive the result of composite Higgs formation from a Dirac pair assuming no composite of Majorana pairing, and vice versa. Assuming a particular form of composite formation, an effective field theory analysis is in principle capable of determining the existence of nontrivial vacuum and the corresponding condition on model parameters. Such results

from Ref.[9] agree with those we obtain from a gap equation analysis [7]. However, such analysis for the HSNJL model has not been available. More importantly, the approach is not considered more powerful in resolving the question of under what condition the model will produce the Dirac or Majorana pair composite as the symmetry breaking Higgs. Actually, the composite can be something in between the Dirac or pure Majorana cases considered. The nontrivial fermion/superfield masses generated may have, in principle, mass eigenstates as any linear combination of $\Phi_{+}$and $\Phi_{-}$. At this point, we actually do not see a way to resolve the problem within the effective field theory approach. Our gap equation approach, in principle, certainly can answer the question. We obtain the full gap equation for the generic superfield mass matrix [10]. It is taking more hard work to extract explicit solution information though.

\section{Acknowledgments}

Y.-M.D.,G.F., and O.K. are partially supported by research Grant No. NSC 99- 2112M-008-003-MY3, and G.F. is further supported by Grants No. NSC 100-2811-M-008-036 and No. NSC 101-2811-M-008-022 of the National Science Council of Taiwan. D.-W.J. is 
supported by NRF Research Grant No. 2012R1A2A1A01006053 of Korea.

[1] Y. Nambu and G. Jona-Lasinio, Phys. Rev. 122, 345 (1961); Phys. Rev. 124, 246 (1961).

[2] Y. Nambu, Enrico Fermi Institute Report No. 89-08 (1989).

[3] V. Miransky, M. Tanabashi, and K. Yamawaki, Mod. Phys. Lett. A 4, 1043 (1989); Phys. Lett. B 221, 177 (1989); W.J. Marciano, Phys. Rev. Lett. 62, 2793 (1989); Phys. Rev. D 41, 219 (1990); W. A. Bardeen, C. T. Hill, and M. Lindner, ibid. D 41, 1647 (1990).

[4] T.E. Clark, S.T. Love, and W.A. Bardeen, Phys. Lett. B 237, 235 (1990).

[5] M. S. Carena, T. E. Clark, C. E. M. Wagner, W. A. Bardeen and K. Sasaki, Nucl. Phys. B369, 33 (1992).

[6] D. W. Jung, O. C. W. Kong and J. S. Lee, Phys. Rev. D 81, 031701 (2010).

[7] G. Faisel, D. W. Jung, and O. C. W. Kong, J.High Energy Phys. 01 (2012) 164.

[8] W. Buchmúller and S. T. Love, Nucl. Phys. B204, 213 (1982).

[9] W. Buchmúller and U. Ellwanger, Nucl. Phys. B245, 237 (1984).

[10] Y.-M. Dai, G. Faisel, D. W. Jung, and O. C. W. Kong( unpublished).

[11] W.A. Bardeen, C.N. Leung, and S.T. Love, Phys. Rev. Lett. 56, 1230 (1986); Nucl. Phys. B273, 649 (1986); Nucl. Phys. B323, 493 (1989). 